\title{
Priority rules for twin automated stacking cranes that collaborate
}

\author{
Héctor J. Carlo*, Fernando L. Martínez-Acevedo \\ University of Puerto Rico - Mayagüez, \\ Industrial Engineering Department
}

Call Box 9000, Mayagüez, PR 00681-9000

Abstract

A recent trend in container ports is to operate twin (i.e., identical non-passing)

Automated Storage Cranes (ASCs) that collaborate to serve storage and retrieval requests from opposite ends of a storage block. Since the ASCs are unable to pass each other, there is an exchange point that serves as a temporary storage location so that one crane can start a request and leave it to the other crane to complete it. In this paper fourteen twin ASC priority rules are proposed and evaluated via simulation with respect to the makespan. The priority rules are then compared to the optimal priority, which is obtained using a Branch and Bound algorithm. Results from an exhaustive simulation study suggests that the best priority rules are LonRem and RemReq, which gives priority to the ASC with the longest remaining estimated workload or to the ASC with the most number of remaining requests, respectively, yielding solutions that are on average $1.3 \%$ from the optimal makespan. It is also found that best two-rule combination is LonRem and PriC2 (the latter gives priority to the seaside ASC). Besides their immediate practical use, the results of this paper are also useful when evaluating candidate solutions for the problem of assigning and scheduling requests in the twin collaborating ASCs system. This study is the first to compare priority rules for collaborating twin ASCs and quantify the impact of the priority rule selected.

Keywords: container terminal; automated stacking cranes; priority rules; simulation

(*) Corresponding author. hector.carlo@upr.edu. 1+787-832-4040 x-3105 


\title{
Priority rules for twin automated stacking cranes that collaborate
}

\begin{abstract}
A recent trend in container ports is to operate twin (i.e., identical non-passing) Automated Storage Cranes (ASCs) that collaborate to serve storage and retrieval requests from opposite ends of a storage block. Since the ASCs are unable to pass each other, there is an exchange point that serves as a temporary storage location so that one crane can start a request and leave it to the other crane to complete it. In this paper fourteen twin ASC priority rules are proposed and evaluated via simulation with respect to the makespan. The priority rules are then compared to the optimal priority, which is obtained using a Branch and Bound algorithm. Results from an exhaustive simulation study suggests that the best priority rules are LonRem and RemReq, which gives priority to the ASC with the longest remaining estimated workload or to the ASC with the most number of remaining requests, respectively, yielding solutions that are on average $1.3 \%$ from the optimal makespan. It is also found that best two-rule combination is LonRem and PriC2 (the latter gives priority to the seaside ASC). Besides their immediate practical use, the results of this paper are also useful when evaluating candidate solutions for the problem of assigning and scheduling requests in the twin collaborating ASCs system. This study is the first to compare priority rules for collaborating twin ASCs and quantify the impact of the priority rule selected.
\end{abstract}

Keywords: container terminal; automated stacking cranes; priority rules; simulation

\section{Introduction}

With the globalization of the economies the freight volume handled at container ports has increased precipitately. As a consequence, most large container ports worldwide have been eager to implement new automated technologies to increase their efficiency in order to maintain their level of competitiveness. One such automated technology is Automated Stacking Cranes (ASCs), which are automated rail-mounted gantry cranes used in the container terminal's storage yard (Gottwald, 2015).

ASCs can be thought of as a second generation of the traditional rubber-tyred gantry cranes (RTGCs) described in Murty et al. (2005). Different from RTGCs, ASCs do not 
require human operators; resulting in lower operational cost, faster speeds, with less container handling errors. On the other hand, ASCs have less flexibility than RTGCs as their movement is limited to the area between the rails. Furthermore, the storage yard layout when using ASCs is typically very different from the one using RTCGs. The former typically has ASCs traveling perpendicular to the berth (European layout), while the latter has RTGCs traveling parallel to the berth (Asian layout). The reader is referred to the overview paper of Carlo et al. (2014) for a discussion and comparison of these layouts.

Several ASC configurations have been implemented in practice. The simplest configuration is a single-ASC used in most European ports. The existing two-crane configurations are the dual and twin configuration. In the dual configuration two different sized ASCs that do not share their rails operate in the same region. The design is such that a smaller ASC is able to go underneath the larger ASC as long as the hoist of the larger crane is all the way up. On the other hand, the non-passing crane configuration simply has two identical ASCs that share a rail. Hence, it is impossible for them to pass each other. For a description and comparison of these ASC configurations the reader is referred to Carlo and Vis (2009). A fourth configuration known as a triple-crane has two smaller twin ASCs and one larger ASC able to pass the others (Dorndorf and Schneider, 2010). In this paper we study a twin ASC configuration such as the one shown in Fig. 1.

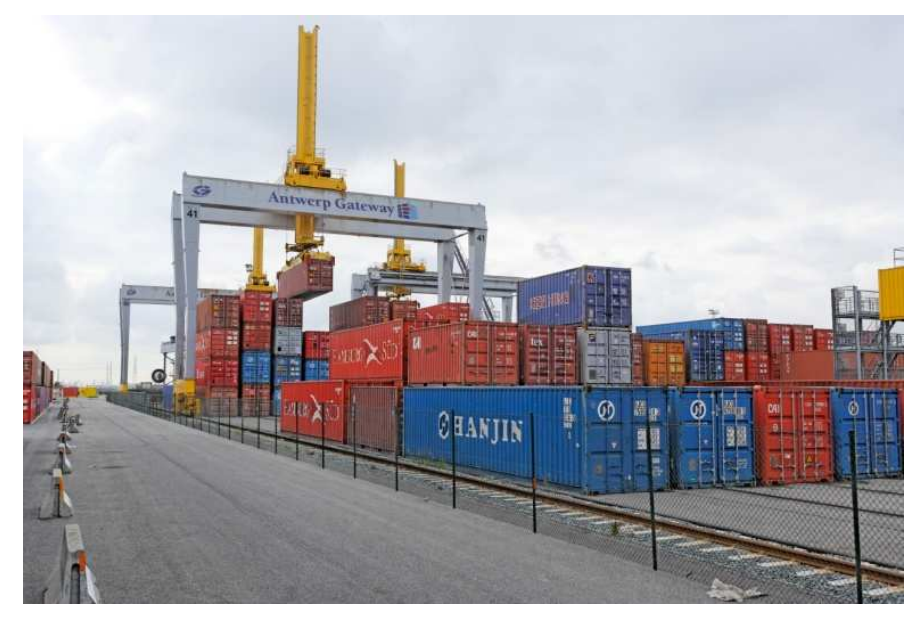

Figure 1: Two Non-passing (twin) ASCs (Gottwald, 2015)

When using ASCs, the storage yard is typically partitioned into storage blocks as shown in Fig. 2. Each block is typically composed of 6-8 lanes (rows), and 20-40 storage bays. In addition, containers are typically stacked 4-6 tiers high. The pick-up and deposit 
points for storage yards using ASCs are at both ends of the block, namely the seaside and the landside. Hence, each block may receive storage or retrieval requests for the seaside or the landside. On the seaside, the ASCs typically pick-up and deposit containers from Automated Guided Vehicles (AGVs) in an automated manner, whereas on the landside they interact with trucks or straddle carriers in a semi-automated manner for safety purposes.

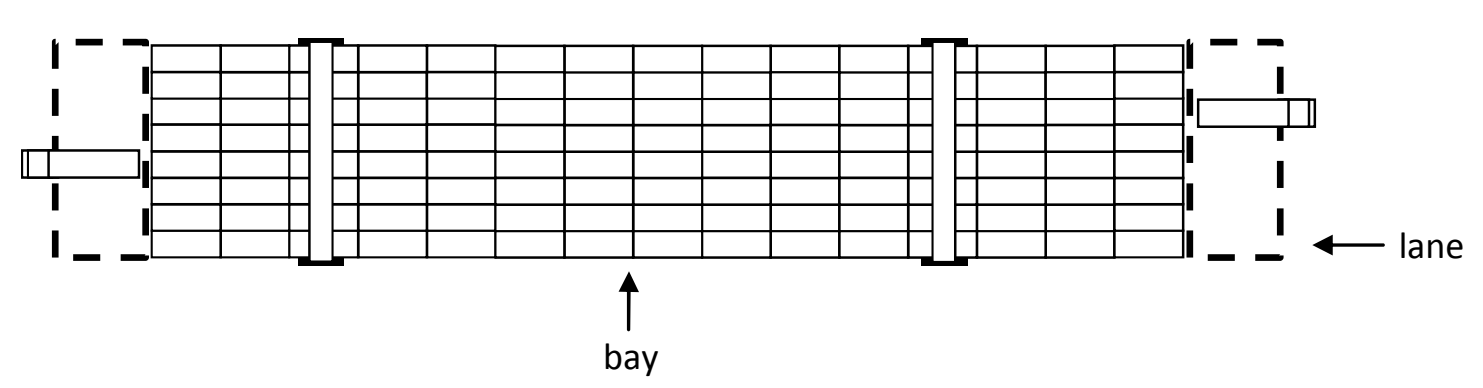

Figure 2: Top-view of a typical storage yard block with twin ASCs in a European layout.

In practice, the twin ASC configuration is implemented without allowing the ASCs to collaborate. In other words, the two ASCs operate in the same storage block, but all requests related to the seaside are handled by one of the ASCs (i.e., the seaside ASC) and all requests related to the landside are handled by the other ASC (i.e., the landside ASC). In this implementation, interference between the ASCs can be easily handled by carefully scheduling the requests. An alternative to this implementation is to allow the twin ASCs to collaborate by defining an exchange point where an ASC may deposit a container for the other ASC to complete the request. This implementation is particularly useful when containers need to be stored or retrieved from a location that is far from its pick-up or deposit point. It also allows for an ASC to share the work when unbalanced, which would ultimately minimize the total makespan. Unfortunately, since the collaborating twin ASCs require an exchange point, the interferences between the ASCs are more complex than in the non-collaborating case. Hence, careful consideration must be given to determining the best priority rules for this implementation.

This paper focuses on evaluating priority rules for the collaborating twin ASC configuration with respect to the makespan, assuming that the assignment of requests to ASCs and their respective schedules are given. Fourteen priority rules are introduced and compared to the optimal priority rule in an exhaustive simulation experiment. The goal of this study is to determine which priority rule (or combination of priority rules) is preferable for different experimental conditions designed to emulate the real-life situations faced in a container terminal. These priority rules are designed to be easily 
implemented under the actual container terminal operational conditions. The remainder of the paper is organized as follows. Section 2 discusses the relevant existing literature on the problem. Section 3 describes the problem and relates it to other decisions for collaborating twin ASCs and presents a mathematical model for the problem. Section 4 presents a sample problem. Section 5 presents a the proposed priority rules. Section 6 describes the proposed Branch and Bound scheme for finding the optimal priorities. Section 7 describes the simulation experiments and results. Lastly, Section 8 includes the conclusions and further research.

\section{Literature Review}

The problem setting in this paper may be generalized as having two identical nonpassing material handling equipment (MHE) that share a travel path and work in the same area serving retrieval and storage requests from opposite sides of the area. The MHE may collaborate by passing loads from one MHE to the other via an exchange point. Unfortunately, there is a dearth of publications considering this setting. Only three papers, all from the container terminals literature, study a setting similar to the one discussed in this paper: Zhou and Wu (2009), Park et al. (2010), and Dorndorf and Schneider (2010).

Zhou and $\mathrm{Wu}$ (2009) focuses on finding the schedule and the exchange point for two collaborating twin ASCs that retrieve a containers from a particular storage bay to the seaside input/output (I/O) point of the block. It is assumed that the ASCs travel at different speeds when empty or full and that all containers must be handled by both ASCs sequentially by using an exchange point. The paper presents necessary and sufficient conditions to determine if all requests can be served without violating their due dates. Based on these conditions, optimal algorithms are provided to minimize the maximum tardiness, the number of tardy jobs, makespan, and weighted completion time. The main differences between Zhou and $\mathrm{Wu}$ (2009) and this paper are: (1) that they only consider retrieval requests from a particular storage bay to the seaside I/O point so the ASCs are collaborating to transport containers from the same place and in the same direction; (2) that the sequence in which the ASCs will serve the jobs is left as a decision; and (3) that the exchange point is considered as a decision.

Park et al. (2010) study the real-time scheduling for a twin ASC system. Collaboration occurs through rehandling and reposition operations, which are considered as optional jobs not explicitly requested. A mathematical formulation and two heuristics (greedy and look-ahead) are proposed and compared. Interference between the ASCs is resolved assigning priority to the seaside ASC for the greedy heuristic, and to the ASC whose current work is positioned earlier in the list of requests assigned to the ASC. 
Dorndorf and Schneider (2010), study a triple-ASCs system composed of two identical twin ASCs and a third larger ASC that may overtake the twin cranes. In their system, yard crane interference occurs between the two twin ASCs and between the twin ASCs and the larger crane whenever the latter lowers its spreader to serve a request. Given a set of storage, retrieval, and reshuffling requests with time windows from the seaside and landside, the problem is to determine which ASC will serve each request and in which sequence. The objective is to maximize the productivity of the triple-ASC system while preventing delays at both ends of the block. The system studied assumes that twin ASCs collaborate in an asynchronous manner. In other words, containers may be handled by both twin ASCs by placing containers in an exchange point. However, it is assumed that the twin ASCs will not place and retrieve a container in the exchange point in the same planning period. The problem is solved dynamically by optimizing the assignment and sequencing of requests whenever a request is completed or a new one arrives. A beam search approach is used for the request-to-ASC assignment. Given request-to-ASC assignment, Branch \& Bound is used to find the optimal routing, considering ASC interference. The main difference between Dorndorf and Schneider (2010) and our work is that precedence constraints that involve the exchange point are not considered in their paper as they separate storing and retrieving containers to the exchange point into different planning periods.

Most twin MHE research does not consider collaboration. Among non-collaborating twin MHE research very few assume that the MHEs travel in the same path and have I/O points at opposite ends, as with the twin ASCs system studied. Erdogan et al. (2014) and Boysen et al. (2015) study the problem of scheduling twin robots that share a travel path and have I/O points on opposite sides. The problem in Erdogan et al. (2014) and Boysen et al. (2015) is operationally identical to the one faced in automated container terminals with twin ASCs and vastly studied in the literature (e.g., Li et al., 2009; Dell et al., 2009; Gharehgozli et al., 2015). Although the setting of these papers is similar to the one considered in this work, the fact that they do not consider collaboration between the MHE significantly simplifies the problem (e.g., precedence constraints for collaborations are not considered).

It is worth mentioning that we are not aware of any operational container terminal currently using a twin ASC system that allows collaboration between the ASCs. Personal communications with several terminal managers suggest that the main reason for not allowing the ASCs to collaborate is because the system operation becomes more complex. Ironically, since the ASC systems are (semi-) automated, the additional system complexity required for the collaboration would be handled by the automated ASC scheduling system software. The ultimate goal of this study is to provide practical solutions for a sub-problem of the twin ASC scheduling problem, namely how to 
prioritize the ASCs when interference occurs, in an effort to facilitate collaboration between twin ASCs in practice.

For an overview and classification of the literature regarding container terminal storage yard equipment and operations the reader is referred to Carlo et al. (2014).

\section{System and Problem Description}

In order to properly describe the system under study, we divide this Section into three Sub-sections. Sub-section 3.1 provides an overview and the practical motivation for the problem. Sub-section 3.2 presents the problem as a mathematical model; whereas Subsection 3.3 describes a graphical representation to solution of the problem.

\subsection{Overview of the Problem}

Figure 3 depicts the system under study, which is composed of two collaborating twin ASCs, $C_{1}$ and $C_{2}$, who serve storage and retrieval requests from both the seaside and landside, respectively. Namely, $C_{l}$ is the ASC closer to the seaside, while $C_{2}$ is the one closer to the landside. The I/O points for the ASCs are in opposite ends of the block. Since the ASCs are unable to pass each other, there is an exchange point that serves as a temporary storage location so that one crane can start a request and leave it to the other crane to complete it. The ASCs are allowed to operate using a dual-command (dualcycle) strategy. A minimum safety distance $(\delta)$ between the ASCs must be observed at all times. The problem setting is characterized in the literature as a twin ASC system (with collaboration), which may found in container terminals with a European layout (Carlo et al., 2014). 


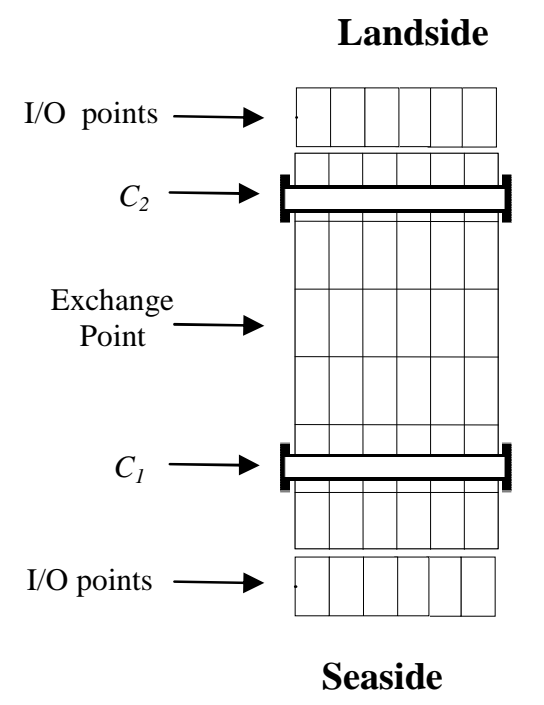

Figure 3: Collaborating Twin ASCs System

The ultimate problem that this research stream intends to solve is the twin ASC assignment and scheduling problem. This problem includes (1) how to assign a list of requests to twin ASCs, (2) how to split the requests among the ASCs, and (3) how to schedule the requests for each ASC. Given the complexity of the twin ASC assignment and scheduling problem, a natural strategy is to efficiently generate basic feasible schedules for each ASC to serve the requests. These schedules would then be evaluated to incorporate the delays caused by the interference between the ASCs. However, in order to compute the delays caused by interference between the ASCs one must first determine how to resolve interferences. Unfortunately, finding the optimal ASC priority for a set of feasible crane schedules would require evaluating $2^{k}$ possible priority assignments, where $k$ is the number of times the cranes interfere - which is unknown before solving the problem. Clearly, this method would be impractical as a sub-routine for any meta-heuristic search procedure. Hence, this study focuses on efficiently solving the sub-problem of determining how to assign priority among two collaborating twin ASCs given the sequence in which each ASC will serve the requests. In practice, container terminals prefer to use simple and practical rules (i.e., rules of thumb) that lead to good solutions, over the optimal solution derived by some black box. Hence, the this paper seeks to introduce and compare fourteen simple ASC priority rules to handle interference between the cranes, given the sequence of storage and retrieval requests for both ASCs.

The underlying ASC assignment and scheduling problem becomes even more complex if we allow the ASCs to collaborate. When the ASCs collaborate one needs to 
add precedence constraints to those requests that are split among the two ASCs (i.e., a split request may not be retrieved from the exchange point until it has been stored there by the other ASC). Furthermore, some of the ASC schedules become infeasible as the ASCs block each other (i.e., deadlock) when both are waiting to retrieve a container the other crane has not yet placed in the exchange point. Hence, it is important to take into consideration how requests that are split among the two cranes are handled.

Throughout this study it is assumed that (storage and retrieval) requests have already been assigned to the ASCs and that the sequence in which the requests will be served for each crane is known and feasible (i.e., it does not result in a deadlock). Further, the storage or retrieval location of each requests, as well as the corresponding I/O point, are assumed given. The performance metric used to compare the priority rules is the makespan (i.e. the maximum completion time of both ASCs) to serve a list of requests. In practice this list of requests would correspond to the workload for a given time period (e.g., one hour worth of requests). The objective of this paper is to empirically identify the best priority rules for resolving interference between the ASCs and to quantify their performance with respect to the optimal ASC priority. The results from this paper will allow future researchers to quickly evaluate candidate solutions for the ASC assignment and scheduling problem. The results are also important for container terminal managers who prefer to implement simple and understandable decision rules in their operations, at the expense of some sub-optimality of the results.

\subsection{Mathematical Model}

A discretized-time mixed integer model for the problem using a similar structure than the model in Dorndorf and Schneider (2010) is presented below. In our problem, two identical ASC cranes collaborate to serve a set of (storage and retrieval) requests in order to minimize the makespan. It is assumed that the requests have already been assigned to one of the cranes and the sequence in which the requests are to be served is also known. Since the ASCs collaborate, storage and retrieval requests may be split among the cranes, creating precedence requirements. For example, a storage request may be started by one crane and finished by the other crane, by temporarily storing it in an exchange point assumed to have infinite capacity. The way requests are split and assigned is also assumed known in our problem. Lastly, the model assumes that time may be discretized and that cranes have infinite acceleration and deceleration (i.e., they travel at a constant full velocity). The mathematical model divides requests into actions that require some stationary processing time. For example, a storage request has two actions, pickup the container from the current location and deposit container to the specified storage location. Let, 
Sets:

$C$ - set of cranes, indexed on $c=1,2$

$A_{c}$ - set of actions to be performed by crane $c$, indexed on $k, k^{\prime}=1,2, . .,\left|A_{c}\right|$, where the first and last actions to be performed by each crane are dummy actions at their depots $P_{k}-$ set of actions that serve as predecessors for action $k$, indexed on $k=1,2, . .,\left|A_{c}\right|$ $T$ - set of time intervals, indexed on $t=0,1, . ., T \mid$

\section{Parameters:}

$r_{c k}-$ the $x$-position (i.e., yard bay) where action $k$ of crane $c$ is performed

$p_{c k}$ - stationary processing time of the $k^{\text {th }}$ action of crane $c$

$v_{c}$ - constant velocity of crane $c$

$\delta$ - minimal (safety) distance between cranes

$l$ - yard block length

\section{Variables:}

$x_{c t}-$ crane $c$ 's position along the yard block at time interval $t$, non-negative variable

$s_{c k}-$ start time of the $k^{\text {th }}$ action of crane $c$, non-negative integer variable

$W$ - makespan of the problem, unrestricted variable

$\min \mathrm{W}$

s.t.

$W \geq s_{c\left|A_{c}\right|} \quad \forall c \in C$

$s_{c k}+p_{c k} \leq s_{c(k+1)} \quad \forall c \in C, k \leq\left|A_{c}\right|-1$

$s_{2 k^{\prime}} \leq s_{1 k} \quad \forall k \in A_{c}, k^{\prime} \in P_{k}$

$s_{1 k^{\prime}} \leq s_{2 k} \quad \forall k \in A_{c}, k^{\prime} \in P_{k}$

$x_{c t}=r_{c k} \quad \forall c \in C, k \in A_{c}, t \in\left[s_{c k}, s_{c k}+1, \ldots, s_{c k}+p_{c k}\right]$

$x_{c t}-v_{c} \leq x_{c(t+1)}$

$\forall c \in C, t \in T$

$x_{c t}+v_{c} \geq x_{c(t+1)}$

$\forall c \in C, t \in T$

$x_{2 t}-x_{1 t} \leq \delta$

$\forall t \in T$

$0 \leq x_{c t} \leq l$

$\forall c \in C, t \in T$

$s_{c k} \in \mathrm{N}_{0}$

$\forall c \in C, k \in A_{c}$

The objective function is to minimize makespan, computed as the maximum of the completion time between the cranes in constraint set (1). Constraint set (2) ensures that the start time of an action occurs after the completion of the preceding one. Constraint sets (3) and (4) are used to enforce split requests are delivered to the exchange point before picking them up. Constraint set (5) ensures that the crane is at the correct position to perform the required action. This constraint set is considered conditional in that it contains a decision variable with an index that depends on the value of a variable - the 
reader is referred to Dorndorf and Schneider (2010) a discussion of how to handle conditional constraints. Constraint sets (6) and (7) limit the travel position of the cranes in time. Constraint set (8) ensures that a minimal safety distance is kept between the ASCs at all times. Lastly, constraint sets (9) and (10) declare the types of decision variables.

Although it does not appear explicit in the mathematical model, since the assignment of requests to cranes and the sequence of each crane is given, the only optimization decision left is which crane gets priority when they interfere with each other. Hence, this paper focuses on analyzing simple priority rules to solve the undelaying problem. Subsection 3.3 describes the problem with a graphical representation as a basis to develop the proposed priority rules.

\subsection{Graphical Representation to Solutions}

Priority rules are only required when the sequence followed by the cranes creates a situation where the cranes interfere with each other. Therefore, to prevent interference between ASCs one needs to track the location of each ASC throughout time. For this purpose we use a graphical representation form to identify interferences between ASCs. The proposed graphical representation is particularly important in order to simulate the ASC operations.

The basis for the graphical representation is the observation that each storage and retrieval request is composed of four types of movements. For example, a storage request starts by performing an empty travel to the pick-up location, which might be of zero distance in the case the ASC is already in that position. The second movement of a storage request is always a pick-up move, in our case representing the time to move the ASC shuttle to pick-up a container. Then, a full travel is performed to the respective I/O point or exchange point. Lastly, a drop-off is performed to release the container. The retrieval process follows a similar structure. Table 1 presents the four movements for each type of request.

Table 1. Description of ASC movements to serve storage and retrieval requests

\begin{tabular}{|c|l|l|}
\hline Steps & Storage Request & Retrieval Request \\
\hline 1 & $\begin{array}{l}\text { move from current position to pick-up } \\
\text { point }\end{array}$ & $\begin{array}{l}\text { move from current position to retrieval } \\
\text { location }\end{array}$ \\
\hline 2 & pick-up container from pickup point & pick-up container from retrieval location \\
\hline 3 & move with container to storage location & move with container to drop-off point \\
\hline 4 & deposit container at storage location & deposit container at drop-off point \\
\hline
\end{tabular}


We introduce the indices $i$ and $j \in\{1,2,3,4\}$ to respectively represent the specific step of a request being performed on $C_{1}$ and $C_{2}$, as defined in Table 1. Clearly, the type of movement and the state (i.e., full or empty) of the crane in a particular step (e.g., $i=3$ ) differs per type of request. This information is then incorporated into a (continuous) function to represent the position of a crane as a function of time, as shown below.

$f(t)$ Function that represents the position of $C_{1}$ (seaside crane) in time $t$.

$\boldsymbol{g}(\boldsymbol{t})$ Function that represents the position of $C_{2}$ (landside crane) in time $t$.

$f_{i}(t)$ Continuous $i^{\text {th }}$ function $f(t)$ that represents the position of $C_{l}$ in time $t$ for a specific request.

$g_{j}(t)$ Continuous $j^{\text {th }}$ function $\boldsymbol{g}(\boldsymbol{t})$ that represents the position of $C_{2}$ in time $t$ for a specific request.

Figure 4 presents an example the various functions for $C_{1}$ handling a retrieval request. The ordinate axis refers to a bay in the storage block. In the example, the ASC starts at level -2 and moves to level 2 in order to pick-up a container. This linear function in $f(t)$ is referred to as $f_{l}(t)$ because it describes the first step from Table 1. In step 2 the container is picked up using function $f_{2}(t)$. In step 3 the ASC moves to position -5 to release the container at this level in step 4.

\section{Crane position vs. Time}

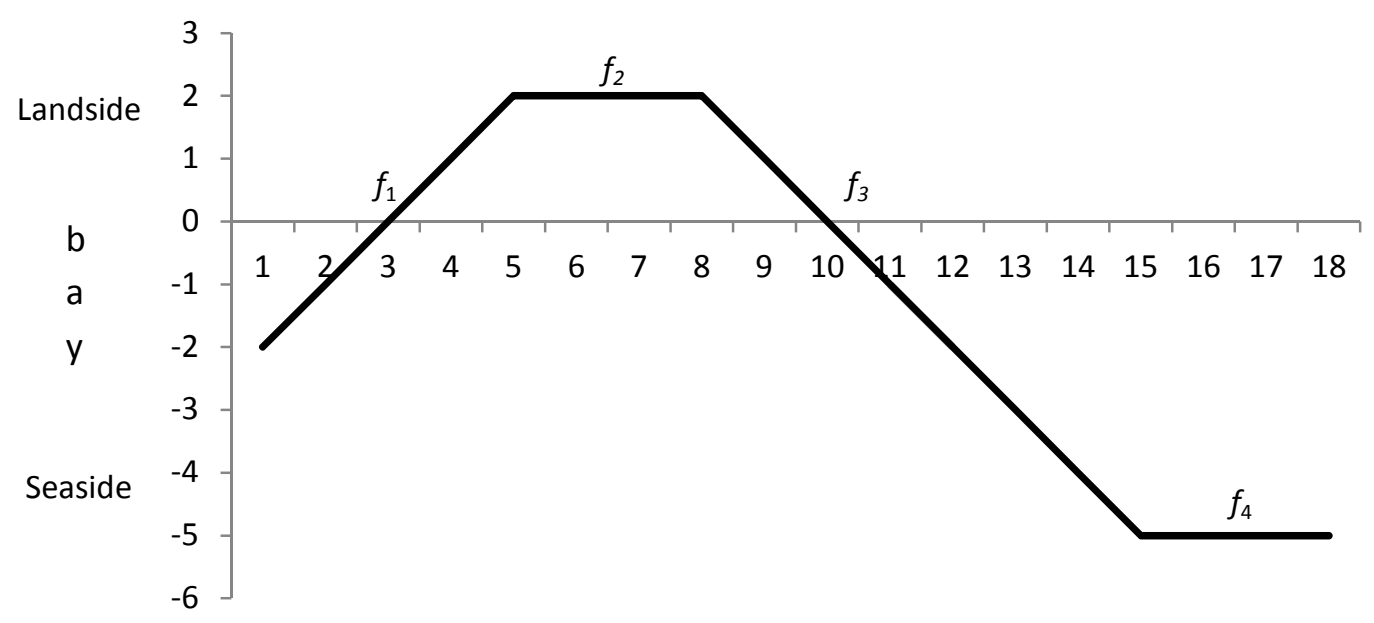

Figure 4: Example of a retrieval request handled by $C_{l}$.

$C_{2}$ would have a movement (and figure) analogous to Fig. 4. Figure 5 depicts a scenario where the $f(t)$ and $g(t)$ are plotted together. From Figure 5 it can be noticed that 
the constraint that the two cranes cannot pass each other is violated. In this case one needs to prioritize the movement of the ASCs. Clearly, in this example, giving priority to $C_{1}$ would result in a lower makespan than giving priority to $C_{2}$.

\section{Crane position vs. Time}

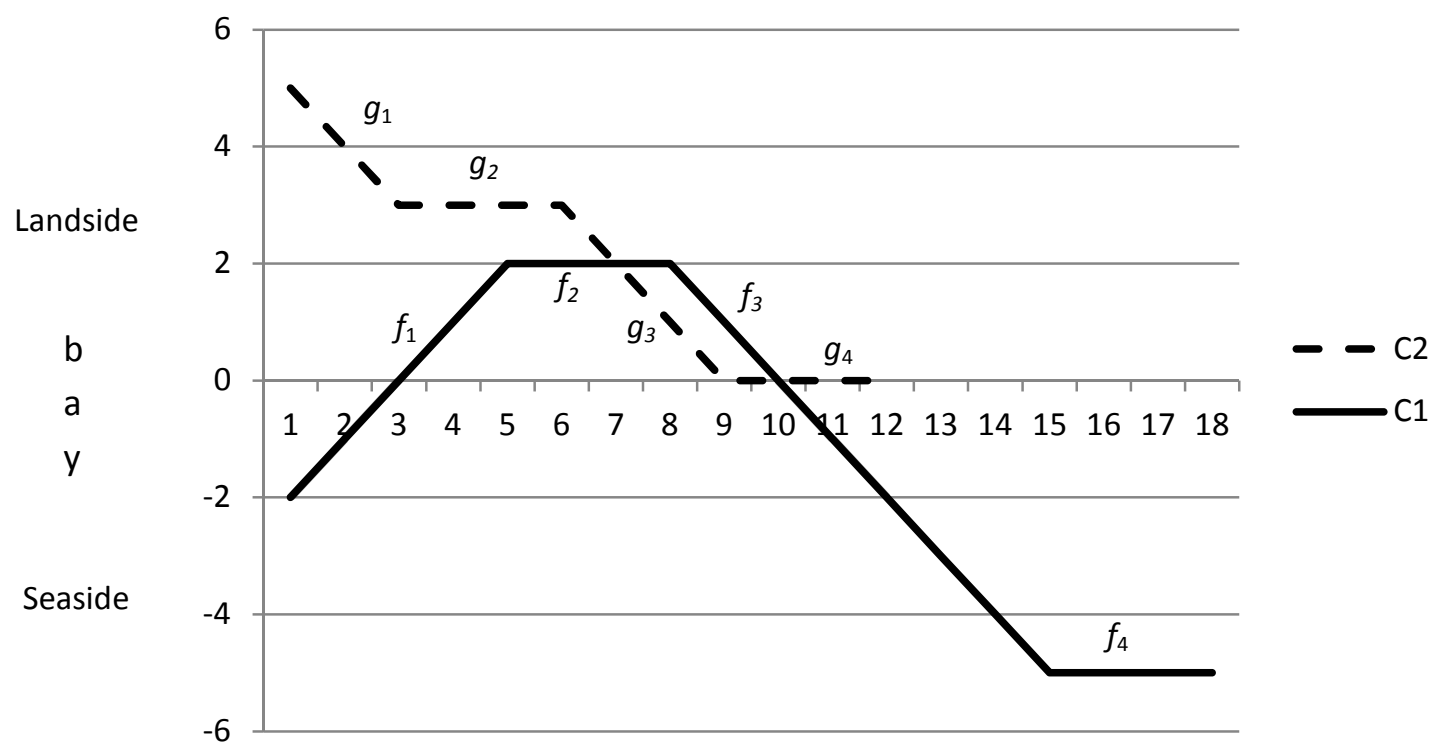

Figure 5: Example of $C_{1}$ and $C_{2}$ handling a retrieval request simultaneously.

One of the benefits of the proposed graphical representation is that mathematically it is very simple to determine if and when interference occurs by finding the intersection of the corresponding piece-wise linear functions. For more details on the graphical representation, the reader is referred to Vis et al. (2010). In Sections 5 and 6, respectively, we present fourteen priority rules and a Brach \& Bound that could be used to resolve interference between the ASCs.

\section{Sample Problem}

To illustrate the problem, consider a 10 request example presented in Table 2. For each crane in the table, the first column indicates the request index (i.e., the sequence in which they will be served), the second column is the description (Descrip.) for the request type and bay associated with the request (e.g., $\mathrm{R}(-3)$ is a retrieval request from bay -3 , and $\mathrm{S}(11)$ is a storage request to bay 11). The third column states the $\mathrm{I} / \mathrm{O}$ associated to the request (i.e., $\mathrm{S}$ for seaside, $\mathrm{L}$ for landside). The fourth column indicates if the request has 
been split. Notice that there are two split requests $\mathrm{R}(-3)$ and $\mathrm{S}(11)$ which are assigned to both ASCs so the 10 requests become 12. Consider for example the second request for $\mathrm{C}_{2}$ in which there is a storage request from the seaside to bay 11 . Since it is a split request, it means that $\mathrm{C}_{1}$ will deliver the container from the seaside to the exchange point, and $\mathrm{C}_{2}$ will move it from the exchange point to bay 11 . Hence, $C_{2}$ must wait for $C_{1}$ to deliver the request before picking it up. The fifth and sixth columns describe the loaded travel associated with the request.

Table 2. Sample problem

\begin{tabular}{|c|c|c|c|c|c|c|c|c|c|c|c|}
\hline \multicolumn{6}{|c|}{$\mathrm{C}_{1}$ (Seaside) } & \multicolumn{6}{|c|}{$\mathrm{C}_{2}$ (Landside) } \\
\hline Index & Descr. & $\mathrm{I} / \mathrm{O}$ & Split & From & To & Index & Descr. & $\mathrm{I} / \mathrm{O}$ & Split & From & To \\
\hline 1 & $\mathrm{R}(-3)$ & $\mathrm{L}$ & Yes & -3 & 0 & 1 & $\mathrm{R}(17)$ & $\mathrm{L}$ & No & 17 & 20 \\
\hline 2 & $\mathrm{R}(10)$ & $\mathrm{S}$ & No & 10 & -20 & 2 & S (11) & $\mathrm{S}$ & Yes & 0 & 11 \\
\hline 3 & $\mathrm{~S}(11)$ & $\mathrm{S}$ & Yes & -20 & 0 & 3 & S (7) & $\mathrm{L}$ & No & 20 & 7 \\
\hline 4 & $\mathrm{~S}(-1)$ & $\mathrm{S}$ & No & -20 & -1 & 4 & $\mathrm{R}(-3)$ & $\mathrm{L}$ & Yes & 0 & 20 \\
\hline 5 & R (19) & $\mathrm{S}$ & No & 19 & -20 & 5 & R (3) & $\mathrm{L}$ & No & 3 & 20 \\
\hline & & & & & & 6 & R (16) & $\mathrm{L}$ & No & 16 & 20 \\
\hline & & & & & & 7 & $\mathrm{R}(6)$ & $\mathrm{L}$ & No & 6 & 20 \\
\hline
\end{tabular}

Figure 6 presents a graphical representation of the optimal solution assuming a $\delta=1$, a velocity of 1 bay per time unit, and a pickup/deposit time of three time units. The optimal solution has a makespan of 265 time units. 


\section{Level vs. Time (Optimal)}

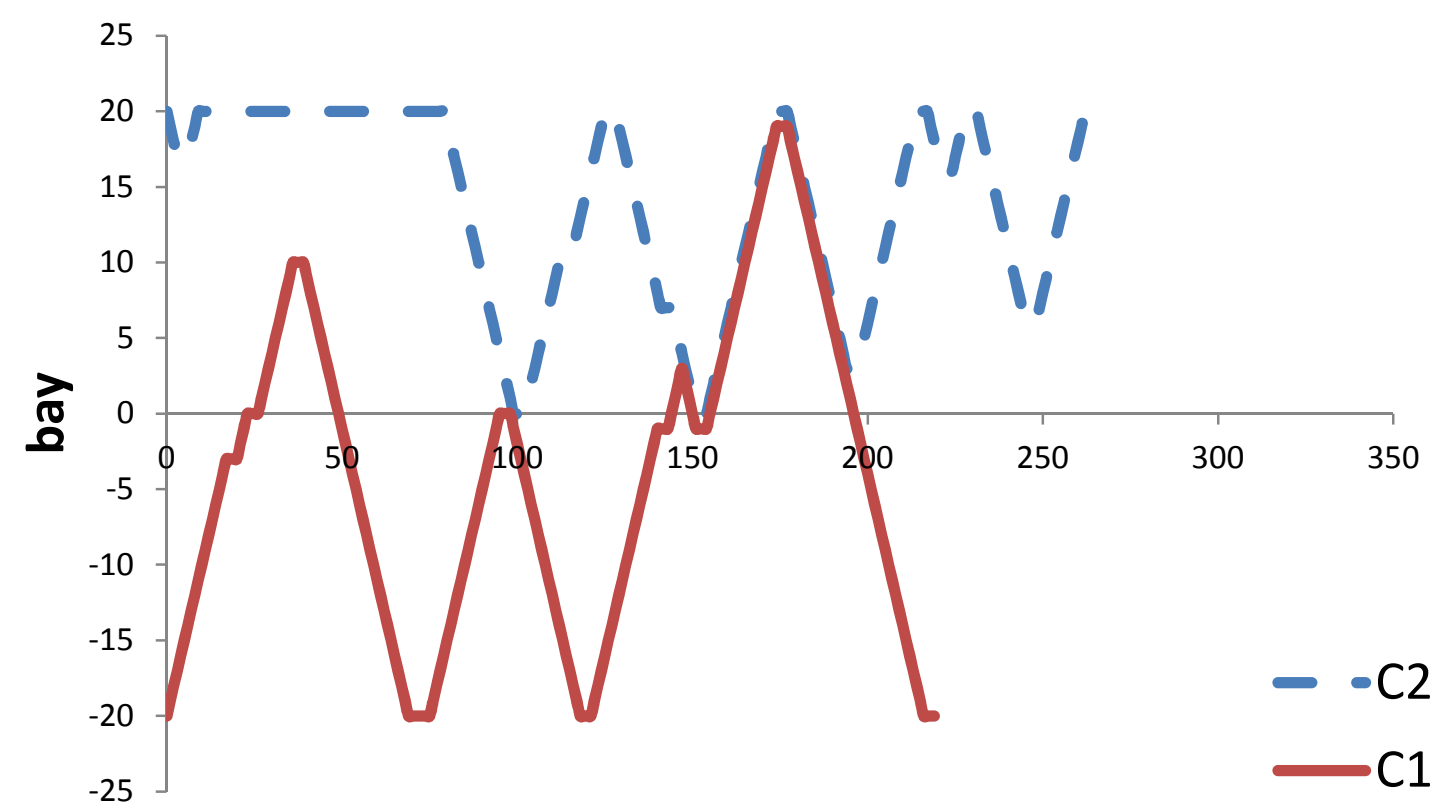

\section{Time}

Figure 6: Graphical representation of optimal solution for example.

From Fig. 6 it can be observed that $\mathrm{C}_{1}$ starts by moving from its $\mathrm{I} / \mathrm{O}$ in bay -20 to bay -3 to serve a retrieval request to the exchange point. Meanwhile, $\mathrm{C}_{2}$ moves from its I/O in bay 20 to bay 17 to retrieve a container back to bay 20. After the first request, $\mathrm{C}_{2}$ finds itself blocked waiting for $\mathrm{C}_{1}$ to complete its third request, which would allow the $\mathrm{S}(11)$ to be executed. During this time, $\mathrm{C}_{1}$ continues performing the prescribed requests. At time $98 \mathrm{C}_{1}$ completes its third request, which unblocks $\mathrm{C}_{2}$. From that moment the ASCs continue moving independently until time 147, in which if both continue their paths they would interfere. As expected by the amount of work remaining, the optimal solution would give priority to $\mathrm{C}_{2}$ at this point. $\mathrm{C}_{1}$ will simply wait for $\mathrm{C}_{2}$ to complete its request, before continuing. The ASCs do not interfere in the remaining of the example. $\mathrm{C}_{1}$ finishes its requests in time 219 , whereas $C_{2}$ in 265 , which is the optimal makespan. Priority rules that gave priority to $C_{1}$ in time 147 yield a makespan of 300 . 


\section{Proposed Priority Rules}

Once interference between the ASCs has been identified using the methodology described in Section 3, a prioritization scheme needs to be executed. In this Section we present fourteen priority rules (P1-P14) for handling interferences between the ASCs.

P1. (PriC1) Crane 1 always has priority. This priority rule was considered in Carlo and Vis (2012) to prioritize two non-collaborating twin cranes with a single I/O point and in the greedy heuristic of Park et al. (2010). PriC1 would be advantageous if most of the requests are either associated with the seaside or assigned to $C_{l}$.

P2. (PriC2) Crane 2 always has priority. This priority rule was also considered in Carlo and Vis (2012). This priority could be advantageous if most of the requests are associated with the landside or assigned to $C_{2}$.

P3. (AdvFun) Upon interference, favor the ASC whose respective function $f_{i}(t)$ or $g_{j}(t)$ has a higher index (or step from Table 1) at the time of the interference. This priority rule was designed to favor the crane closest to completing a request (in terms of the number of movements remaining).

P4. (ShoOri) Upon interference, favor the ASC with the shortest travel time to the origin of the next request (upon completion of the current request). This priority rule seeks to minimize the empty travel time to the next request.

P5. (LonOri) Upon interference, favor the ASC with the longest travel time to the origin of the next request (upon completion of the current request). This priority rule was designed recognizing that a long unproductive (i.e., empty) and unavoidable move needs to be performed, which is less likely to re-interfere with the non-prioritized ASC.

P6. (ShoFin) Upon interference, favor the ASC with the shortest time to finish the current request. The idea behind this priority rule is to minimize the wait time for the non-prioritized crane.

P7. (LonFin) Upon interference, favor the ASC with the longest time to finish the current request. The idea behind this priority rule is to allow longer requests to be prioritized.

P8. (AbsDis) Upon interference, favor the ASC with the shortest travel time to the exchange point. This priority rule seeks to favor the crane that is furthest away from its I/O point. Notice that in order for the ASCs to interfere one must be past the exchange point.

P9. (TotReq) Upon interference, favor the ASC to which most requests have been assigned. This priority rule is based on the premise that the more requests a crane has to serve, the more likely it is to be the bottleneck crane. 
P10. (RemReq) Upon interference, favor the ASC that has more requests left to serve. The spirit of this priority rule is to identify the bottleneck crane and give it priority. It is meant as an improvement over the local search heuristic priority of Park et al. (2010).

P11. (LonTot) At the beginning of the problem, determine the total time each ASC needs to complete its requests (considering all assigned requests) without considering the other crane (i.e., no interference). Give priority to the crane that requires the most time to finish all the assigned requests. Similar to P9 and P10, this priority rule seeks to give priority to the bottleneck crane.

P12. (LonRem) Whenever there is interference between the ASCs, determine the time each ASC would need to complete the remaining requests without considering the other crane (i.e., no interference). This is equivalent to the Priority Longest rule in Carlo and Vis (2012).

P13. (MidCross) Interference between the ASCs is handled by having the ASC that has to cross the exchange point to wait until the other ASC is not in the way. After an ASC crosses the exchange point, it has priority over the other crane until it returns to its side. This priority was introduced by Dell et al. (2009).

P14. (Rand) Interference between the ASCs is handled randomly (i.e., each crane has $50 \%$ probability of receiving priority).

It is important to note that although these priority rules are simple by design, they take into considerations key characteristics of collaborating twin ASC systems, including the fact that some requests (i.e., split requests) need to be handled by both cranes. Also, it is worth mentioning that although these priority rules are designed for minimizing makespan, most of them may be easily adapted for other objective functions.

\section{A Branch \& Bound Algorithm for the Optimal Priority}

The optimal priority (i.e., which ASC gets priority in case of interference) may be obtained by exhaustive enumeration of the $2^{k}$ possible priority assignments, where $k$ is the number of times the ASCs interfere in a given problem instance. In this paper we propose a Branch \& Bound $(\mathrm{B} \& \mathrm{~B})$ algorithm as a more efficient alternative to obtain the optimal priority. In the proposed $\mathrm{B} \& \mathrm{~B}$, every time the two ASCs interfere, two new branches are created (one with Priority to $C_{1}$ and the other with Priority to $C_{2}$ ). A lower bound is obtained for each branch by determining the resulting makespan if all future interference delays and split-requests interactions are ignored. It is interesting to note that although the $\mathrm{B} \& \mathrm{~B}$ is more efficient than exhaustive enumeration, and on average 
relatively fast, the runtime of the algorithm depends on the number of times the ASCs interfere, which is unknown at the beginning of the algorithm. Given that the number of branches to be explored is unknown at the beginning of the problem; our B\&B requires a dynamic structure to create new branches. As in regular B\&Bs, branches are pruned if their computed lower bound is higher than an existing or computed upper bound. Figure 7 presents a flow chart for the proposed $\mathrm{B} \& \mathrm{~B}$. In this figure, $U B$ refers to an upper bound and $U B^{*}$ is the best upper bound found. The lower bound $(L B)$ is computed by serving all remaining requests ignoring interference and interactions between ASCs. 


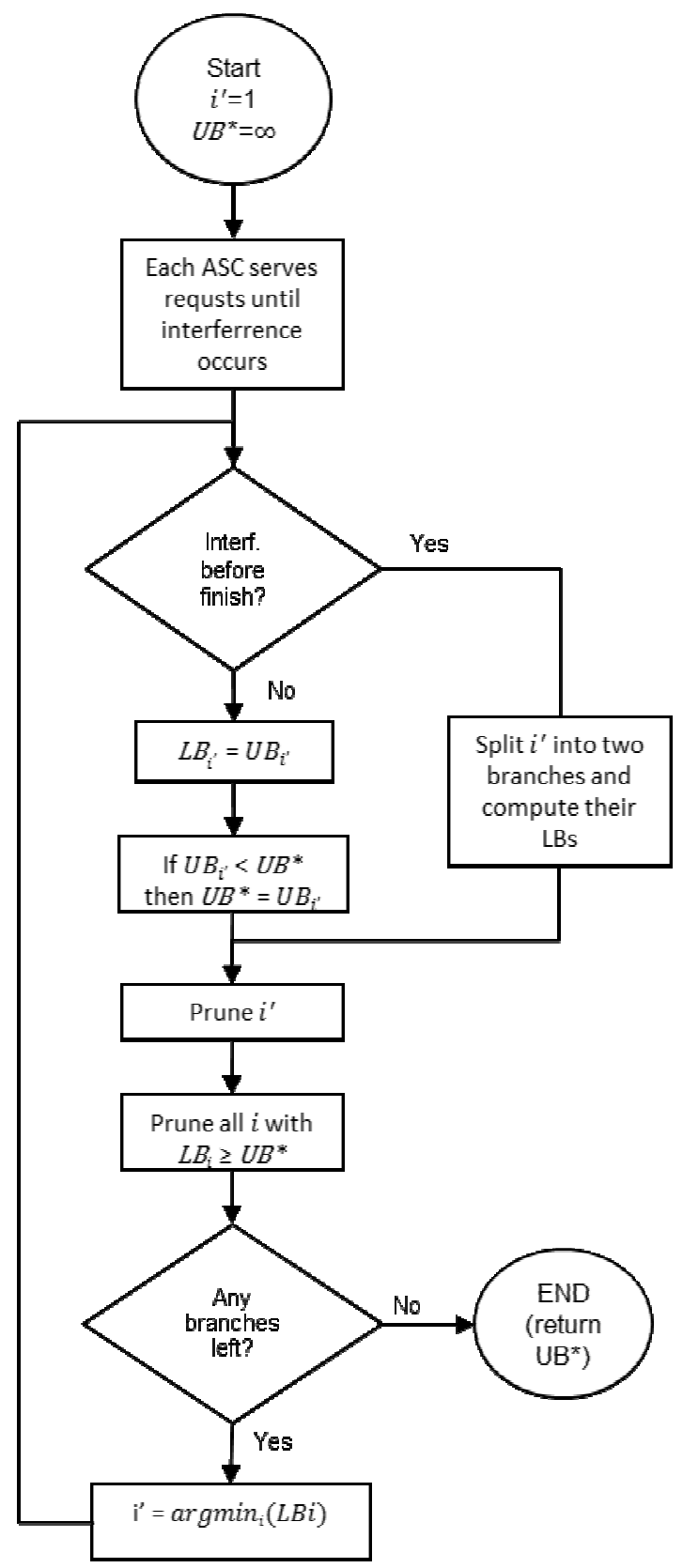

Figure 7: Graphical representation of optimal solution for example.

It is important to remember that the problem addressed in this paper will be a subproblem of a larger optimization problem that seeks to determine how to assign a block of requests to two twin ASCs, how to split the requests among the ASCs, and how to schedule them for each ASC. Clearly, in order to evaluate the fitness of assigning a particular request to an ASC and the ASC schedule, a priority rule needs to be determined. Given the complexity of the overlaying problems, the scope of this research 
is limited to identify simple priority rules for when the ASCs interfere. In the next section the optimal solution from the $\mathrm{B} \& \mathrm{~B}$ described above is used to assess the performance of the proposed priority rules under different experimental conditions.

\section{Simulation}

We use simulation to compare the fourteen proposed priority rules with the optimal priority rule obtained using the $\mathrm{B} \& \mathrm{~B}$ algorithm. In order to speed up the $\mathrm{B} \& \mathrm{~B}$ algorithm in our simulation, the best found upper bound between the 14 priority rules in Section 5 was used as an initial upper bound. Also, for the B\&B implementation we opted for a greedy approach where the most promising branch (i.e., the lowest lower bound) is selected to be explored one more level. This strategy was preferred over a breadth-first strategy given the existence of good upper bounds.

The full factorial design of experiments presented in Table 3 was used to compare the fourteen priority rules under different conditions. The experiment has five factors: number of requests (3 levels); percent of requests that are associated with the seaside (2 levels); percent of requests that are storages (3 levels); percent of requests that are splittable (2 levels); and percent of splittable requests that are actually split (2 levels). The first factor refers to the total number of requests handled between the two ASCs. The second and third factors stipulate the percent of requests associated with the seaside and the percent that are storage requests, respectively. The fourth factor refers to the percent of requests that their storage or retrieval location is further than the exchange point, measured in bays from the (sea or land) side to which they are associated. Notice that when the storage or retrieval location is further than the exchange point, the request may be split among both cranes by using the exchange point (i.e., splittable request). Lastly, the fifth factor defines the percent of those splittable requests (defined in Factor 4) that are actually split. Therefore, if for example there are 50 requests (Factor 1 at Level 3), $25 \%$ are splittable requests (Factor 4 at Level 1), and 50\% are actually split (Factor 5 at Level 2), then we expect on average to have $50 * 0.25 * 0.50=6.25$ split requests. Hence, the average total number of requests for both ASC in our example would be $56.25(=50+$ 6.25 ), as 6.25 of the requests would appear in both cranes. Each factor-level combination in the design of experiments (referred to as a problem instance) represents a potential operational profile (i.e., scenario) faced by container terminals.

Table 3: Design of Experiments

\begin{tabular}{|c|c|c|c|}
\hline Factor & Level 1 & Level 2 & Level 3 \\
\hline Number of Requests & 10 & 25 & 50 \\
\hline
\end{tabular}




\begin{tabular}{|l|c|c|c|}
\hline Percent Requests Sea Side & $75 \%$ & $50 \%$ & - \\
\hline Percent Requests Storages & $75 \%$ & $50 \%$ & $25 \%$ \\
\hline Splittable Requests & $25 \%$ & $50 \%$ & - \\
\hline Percent Splittable that are Split & $25 \%$ & $50 \%$ & - \\
\hline
\end{tabular}

The 72 problem instances from the full factorial experiment in Table 3, as well as the $\mathrm{B} \& \mathrm{~B}$ and the fourteen priority rules were coded in $\mathrm{C}++$. One hundred replicates were run for each instance in a Dell Inspiron personal computer with and Intel Core $i 7$ processor with dual $2.20 \mathrm{GHz}$ processors and $8 \mathrm{~GB}$ of RAM. Considering the fifteen methods compared ( $\mathrm{B} \& \mathrm{~B}$ and fourteen priority rules), the 72 problem instances, and the 100 replicates, a total of 100,800 runs were performed as part of the simulation study.

It was assumed that the storage or retrieval location of the requests was uniformly distributed over 39 storage bays. Furthermore, containers to be retrieved were readily available without reshuffling. The exchange point was located in the middle bay of the storage block and is assumed to have infinite capacity. Two input/output points were also considered at the end of the storage block, as customary for automated ASC systems. The starting location for the two ASCs $\left(C_{1}\right.$ and $\left.C_{2}\right)$ was the seaside and landside, respectively. No specific ending location was enforced for the ASCs. The minimum safety distance between ASCs was assumed to be one distance unit (DU), i.e., $\delta=1$. The total makespan for each instance was recorded assuming that the ASCs travel at a constant velocity of one DU per bay, whereas the pick-up and deposit time was 3 time units. For simplicity, the inter-row movement of the ASCs was not considered in the experiments. This is analogous to stating that the storage block had a single row. The reason for this simplifying assumption is that in practice the inter-bay travel time is much longer than the inter-row movement. Hence, our assumption has minimal impact with respect to ASC travel time under Chebyshev travel.

Unfortunately, creating feasible problem instances is not trivial as $C_{1}$ could be waiting for a split request that $C_{2}$ has not deposited in the exchange point, while $C_{2}$ is also waiting for a split request that $C_{1}$ has not deposited in the exchange point. When the randomly generated problems turned out infeasible, we randomly moved one of the split requests that creates the infeasibility up or down in the corresponding ASC sequence.

As stated earlier, our performance metric to compare the priority rules is makespan (i.e., maximum completion time among both ASCs). It can be said that the makespan in our problem has a fixed and a variable (time) component. The fixed component is the travel time required to complete all requests; which is implicitly given with the assignment of requests to ASCs and the sequence in which they are served by each ASC. On the other hand, the variable component is the travel time incurred as a consequence of the interference and interactions between the cranes. Clearly, the variable component of the makespan is considerably smaller than the fixed component. Furthermore, since 
priority rules only take effect in the variable time component, the response variable used in our experiments is the percent difference between the resulting makespan and the fixed travel time (computed in our lower bound). Representing our results in percent allows us to analyze the instances (which are different in nature) together. For simplicity, however, we will continue to refer to our response variable as makespan.

Table A-1 in the Appendix presents the average percent difference between the makespans and the optimal solution over the 100 replicates for the 72 problem instances. The optimal makespan was computed using the proposed B\&B. It is important to note that in our experiments some replications did not have interference between the ASCs. In these instances all priority rules find the optimal solution by definition. In our experiments, $70.79 \%$ of the runs experienced some interference. The last column of the Table A-1 presents the average number of interferences per instance. The last three rows of Table A-1 present the average and standard deviation over the 72 problem instances, and the number of times each priority rule yield the best makespan among the other (nonoptimal) rules. For each problem instance, the best found priority rule is highlighted in bold.

Table 4 presents a summary of Table A-1, including percent difference from optimal of the average makespan (Ave.), its standard deviation (Stdev), corresponding rank based on the average $\left(\operatorname{Rank}_{A v e}\right)$, average number of times the priority found the optimal solution among all replications (NumOpt), the rank based on the number of times the optimal solution was found $\left(\operatorname{Rank}_{N u m O p t}\right)$, and the percent of instances the priority rule yield the best result from any priority rule $(\%$ Best $)$ and its respective rank $\left(\right.$ Rank $\left._{\text {best }}\right)$. For example, the second column in Table 4 (i.e., Ave.) represents the average (over the 72 instances) percent difference in makespan for each priority with respect to the optimal.

Table 4: Summary of Results by Priority Rule

\begin{tabular}{|l|c|c|c|c|c|c|c|}
\hline Priority & Ave. & Stdev & Rank $_{\text {Ave. }}$ & NumOpt & Rank $_{\text {NumOpt }}$ & $\%$ Best & Rank $_{\text {Best }}$ \\
\hline LonRem & $10.54 \%$ & $7.65 \%$ & 1 & $78.08 \%$ & 1 & $45.33 \%$ & 1 \\
\hline RemReq & $10.69 \%$ & $7.22 \%$ & 2 & $75.96 \%$ & 2 & $29.33 \%$ & 2 \\
\hline LonTot & $13.09 \%$ & $8.01 \%$ & 3 & $72.60 \%$ & 3 & $6.67 \%$ & 3 \\
\hline TotReq & $13.52 \%$ & $7.33 \%$ & 4 & $70.76 \%$ & 4 & $2.67 \%$ & 4 \\
\hline PriC1 & $18.64 \%$ & $10.04 \%$ & 5 & $66.99 \%$ & 5 & $0.00 \%$ & 5 \\
\hline ShoFin & $20.63 \%$ & $12.92 \%$ & 6 & $46.63 \%$ & 9 & $2.67 \%$ & 9 \\
\hline ShoOri & $21.64 \%$ & $14.92 \%$ & 7 & $45.50 \%$ & 10 & $2.67 \%$ & 10 \\
\hline Rand & $22.86 \%$ & $16.08 \%$ & 8 & $49.28 \%$ & 8 & $1.33 \%$ & 8 \\
\hline LonOri & $24.35 \%$ & $17.24 \%$ & 9 & $53.93 \%$ & 6 & $0.00 \%$ & 6 \\
\hline AbsDis & $24.53 \%$ & $21.30 \%$ & 10 & $43.68 \%$ & 11 & $1.33 \%$ & 11 \\
\hline
\end{tabular}




\begin{tabular}{|l|c|c|c|c|c|c|c|}
\hline LonFin & $25.49 \%$ & $18.57 \%$ & 11 & $52.31 \%$ & 7 & $1.33 \%$ & 7 \\
\hline MidCross & $26.08 \%$ & $24.74 \%$ & 12 & $42.88 \%$ & 13 & $0.00 \%$ & 13 \\
\hline AdvFun & $26.56 \%$ & $21.33 \%$ & 13 & $42.14 \%$ & 14 & $1.33 \%$ & 14 \\
\hline PriC2 & $26.80 \%$ & $26.16 \%$ & 14 & $43.42 \%$ & 12 & $5.33 \%$ & 12 \\
\hline
\end{tabular}

In general, all priority rules ran instantaneously, while the $\mathrm{B} \& \mathrm{~B}$ took anywhere from one second to thirty minutes, depending on the instance. By examining the second and third columns in Table 4 one immediately observes that the average performance of all priority rules is between $10.54 \%$ and $26.80 \%$ from optimal. By observing the ranks in columns 4 and 6 of Table 4, it is observed that LonRem and RemReq, respectively, are the best performing priority rules. For instance, if LonRem or RemReq are used as a priority rules, on average, the makespan will within $10.54 \%$ and $10.69 \%$ from optimal, respectively. LonRem and RemReq will find the optimal solution is found in $78.08 \%$ and $75.96 \%$ of the instances, respectively. In fact, a Tukey's Multiple Comparison Analysis with a confidence level of $95 \%$ performed on the data confirms that the optimal solution is statistically better than any priority, yet there is no statistical difference among LonRem and RemReq at the $95 \%$ confidence level. On the other hand, it is found that the worst performing priority rules are MidCross, AdvFun, and PriC2, respectively. It is noted that our experimental design negatively affects PriC2 as the percent of requests that are associated with the seaside was always greater than or equal to $50 \%$; whereas $C_{2}$ is associated with the landside. Hence, when a container terminal experiments more landside requests than seaside requests, because of symmetry, PriC2 would yield the results found for PriCl in our experiments. Lastly, it is interesting to note that six priority rules (including the one from Dell et al., 2009; i.e., MidCross) performed worse than randomly assigning priorities (i.e., Rand), although the statistical test failed to reject the hypotheses that Rand outperformed any of them at the 95\% confidence level. Lastly, if the total (fixed and variable) time is considered in the makespan, LonRem and RemReq would yield solutions within $1.3 \%$ of the optimal total makespan.

As mentioned earlier, the proposed priority rules could be very useful to resolve interference between ASCs when determining how to assign a block of requests to the twin ASCs, how to split the requests among the ASCs, and how to schedule them for each ASC. When used in this context, the proposed priority rules could be used instead of the $\mathrm{B} \& \mathrm{~B}$ to evaluate candidate solutions for the larger optimization problem considering the average optimality gap reported (10.54\% for LonRem). However, given since the optimality gap is still large and given how fast the priority rules are, researchers might prefer to execute two different priority rules in series when evaluating candidate solutions. In this case, the best of the two priority rules would be selected. We considered 
all possible two-way combinations of the priority rules. To determine the performance of each two-priority-rule combination we selected the best performing priority rule from each pair for each run. The results indicate that the best performing two-priority-rule combinations are PriC2-LonRem, LonRem-MidCross, and PriC1-PriC2, respectively. These two-priority-rule combinations were able to yield makespans that were within $3.42 \%, 3.67 \%$, and $3.69 \%$ from optimal, respectively. These results may be directly compared with the best single priority rule (LonRem) which finds solutions within $10.54 \%$ from optimal at the expense of a negligible additional runtime. As expected, combinations containing priority rule LonRem perform well. Interestignly, the best LonRem combinations occurred when it was paired with the worst performing rules. Another interesting finding is that two-priority-rule PriC1-PriC2 performed very well despite not performing that well individually. In general, it may be interpreted that the best performing two-priority-rules are those rules that complement each other best.

\section{Conclusions and Future Work}

This paper is concerned with how to assign priority to collaborating twin ASCs when interference between them occurs. The problem studied is a sub-problem of the assignment and scheduling problem for collaborating twin ASCs which seeks to determine: (1) the requests that are split among the ASCs, (2) the request-to-ASC assignment, and (3) the sequence in which each ASC will serve the requests. In order to evaluate a candidate solution for the larger (and more complex) problem one needs to compute the delays experienced by the ASCs due to interference. In order to compute those delays one needs to determine which crane has priority over the other in case of interference between them. Ideally, priority rules need to be computationally fast, robust, and close to optimal. This study proposes and evaluates fourteen priority rules for resolving interference on a system of twin ASCs that are able to collaborate by passing containers from one ASC to the other via an exchange point. The proposed priority rules were designed so they can be used as rules of thumb in container terminals.

A carefully designed simulation study was used to compare the proposed priority rules under various experimental conditions based on the makespan required to serve a predetermined number of storage and retrieval requests at the seaside and landside of the storage block. A Branch \& Bound (B\&B) algorithm was developed in order to compare the performance of the proposed priority rules with the optimal priority. This study is the first to compare priority rules for twin ASCs and quantify the impact of the priority rule selected.

It is argued that the makespan is composed of a fixed and variable component. The fixed component is the time required to serve all requests if the cranes operated 
independently. The variable time component of the makespan is the time incurred due to interference and blocking of the cranes. Since the priority rules are associated with the variable component of the makespan, we normalize the simulation results to be the percent difference with the lower bound, which ignores interference and blocking. The recommended priority rules based on our results are LonRem and RemReq, which yield a makespan that was on average $10.54 \%$ and $10.69 \%$ from the optimal solution, respectively. However, when considering the total (fixed and variable) makespan, these two priority rules yield average solutions within $1.3 \%$ of the optimal.

Since the priority rules run extremely fast (even on a personal computer), two different priority rules may be run sequentially almost instantaneously, and the one that yields the best makespan is selected. In the case two priority rules are to be used sequentially, the rule combinations PriC2-LonRem, LonRem-MidCross, and PriClPriC2 were the best performing yielding makespans that were within $3.42 \%, 3.67 \%$, and 3.69\% from optimal, respectively.

In general, the proposed priority rules have good performance in terms of solution time and quality. Future work may use these results for evaluating candidate solutions for the complex problem of determining if a request is to be split, assigning requests them to ASCs, and scheduling them. Also, as a next step, it would be appropriate to understand the effect of the location of the exchange point on the priority rules and to optimize its location. Lastly, future work may focus on understanding the effect of different minimum safety distances between the ASCs have on priority rules. 


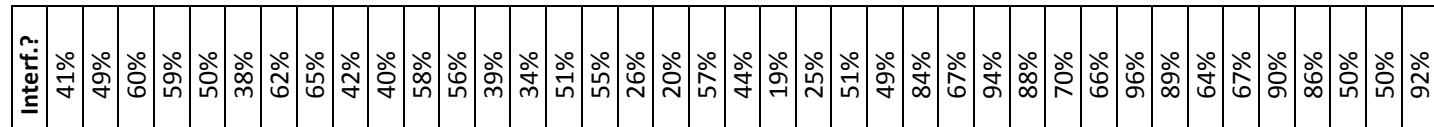

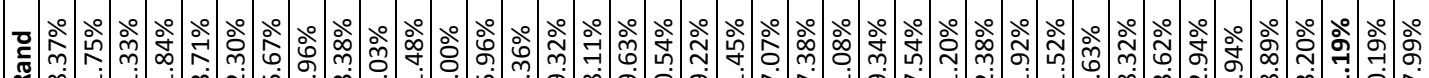

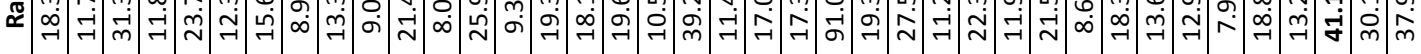

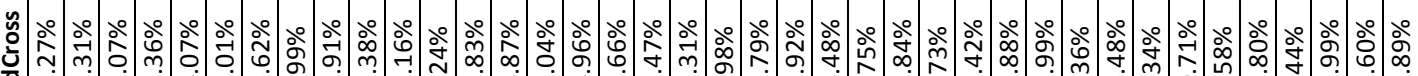

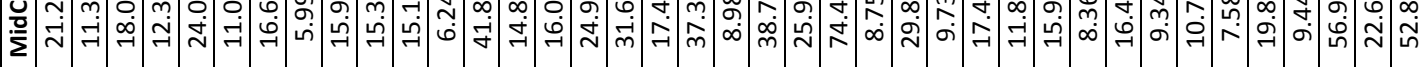

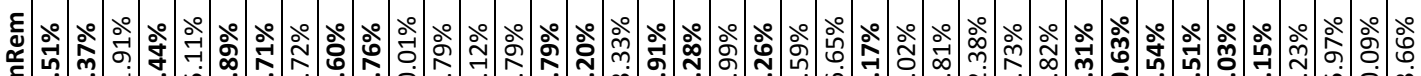

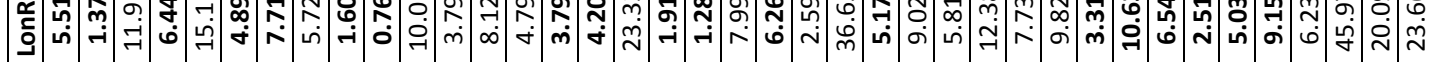

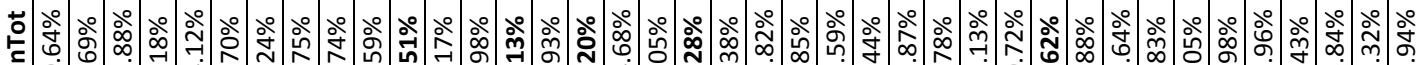

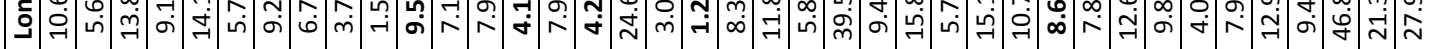

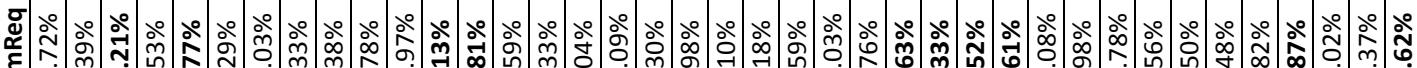

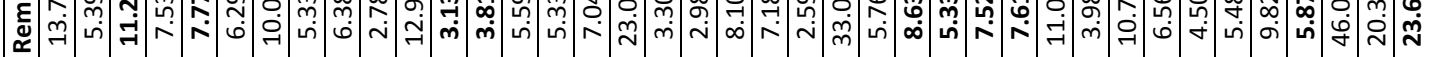

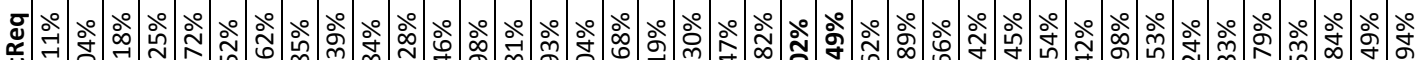
을

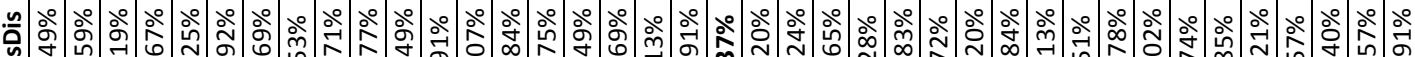

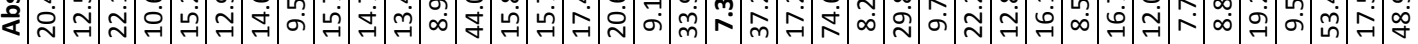

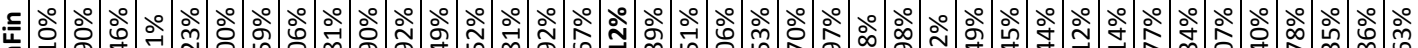
ğ

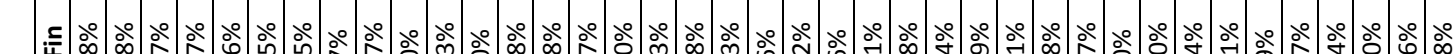

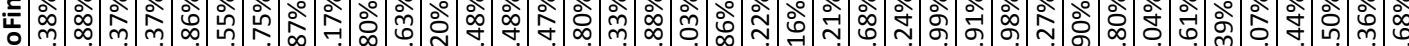
ஸी

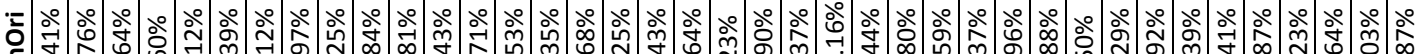
தٔ

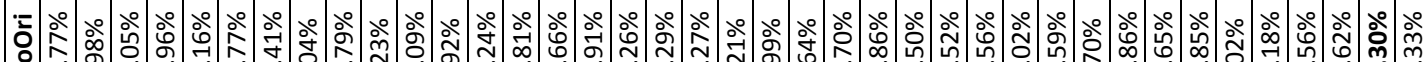

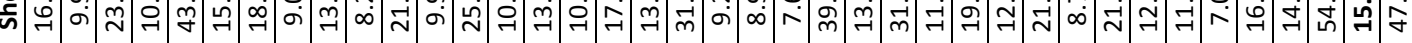

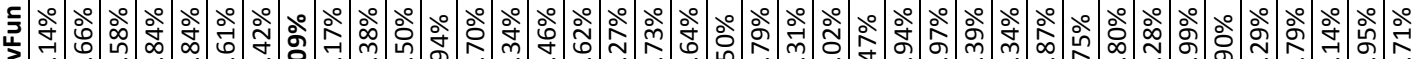

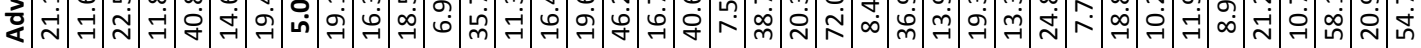

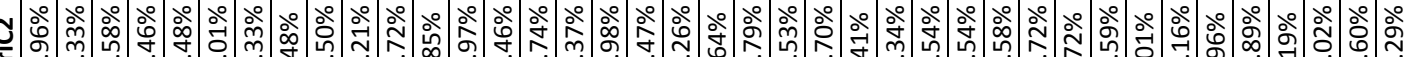
ڤ

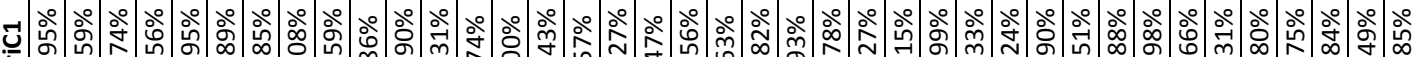

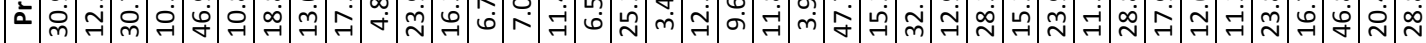

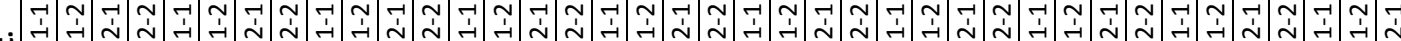

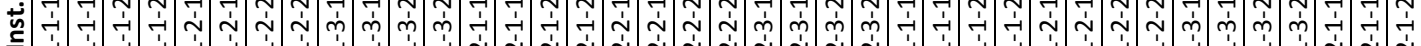

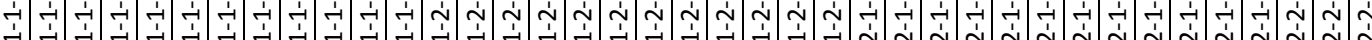




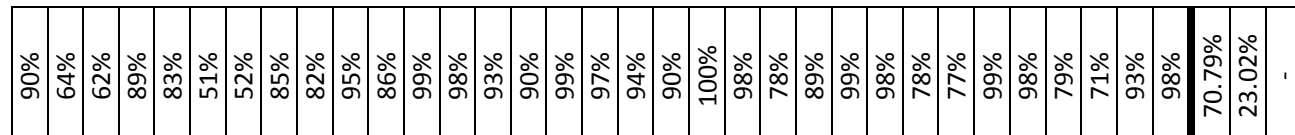

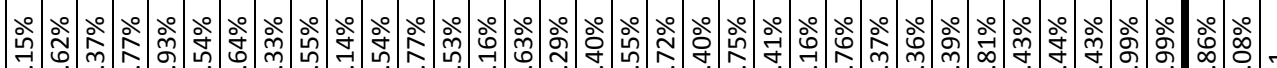

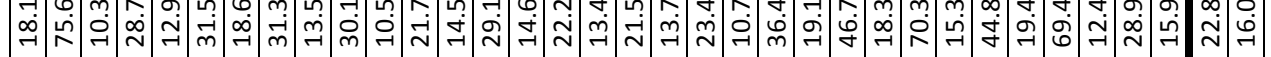

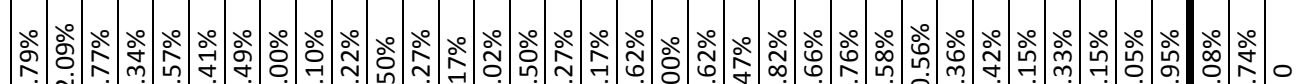

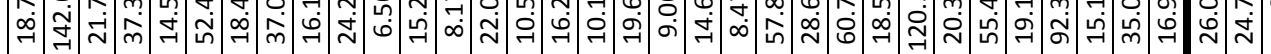

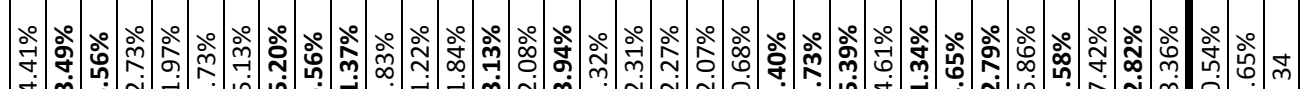

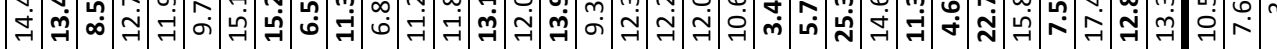

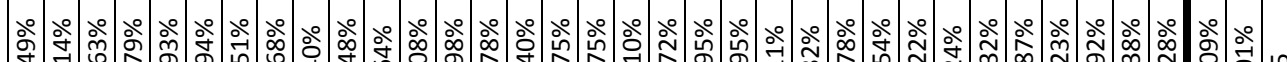
ก

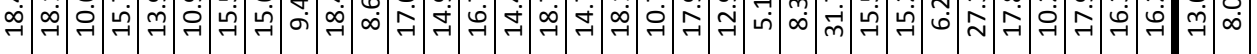

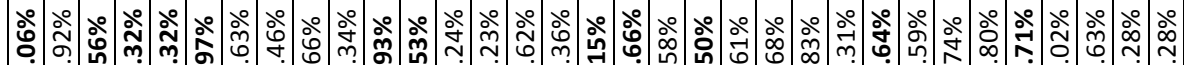
ற்

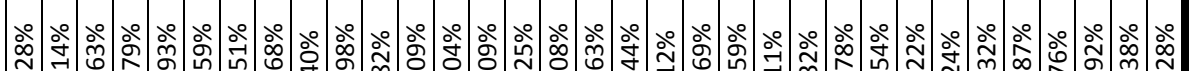

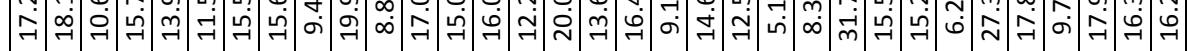

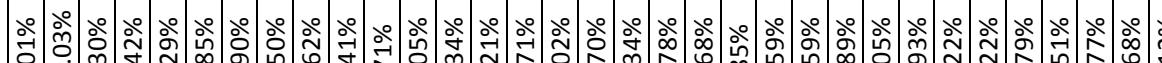

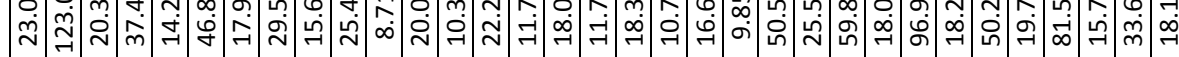

วำ

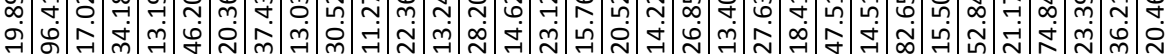

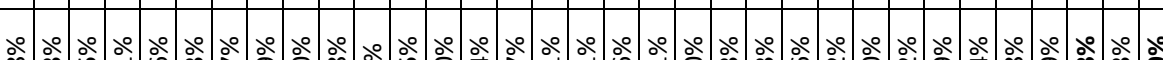

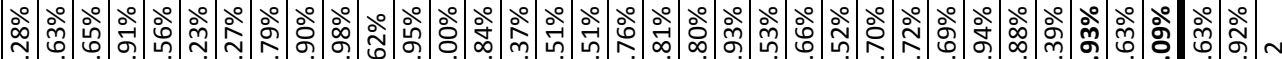

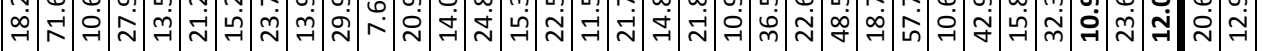

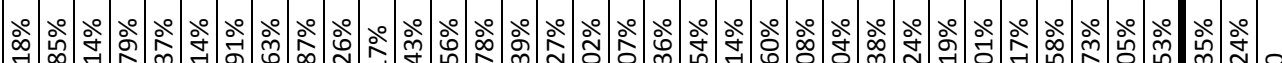

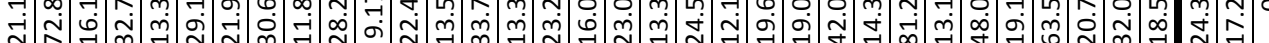

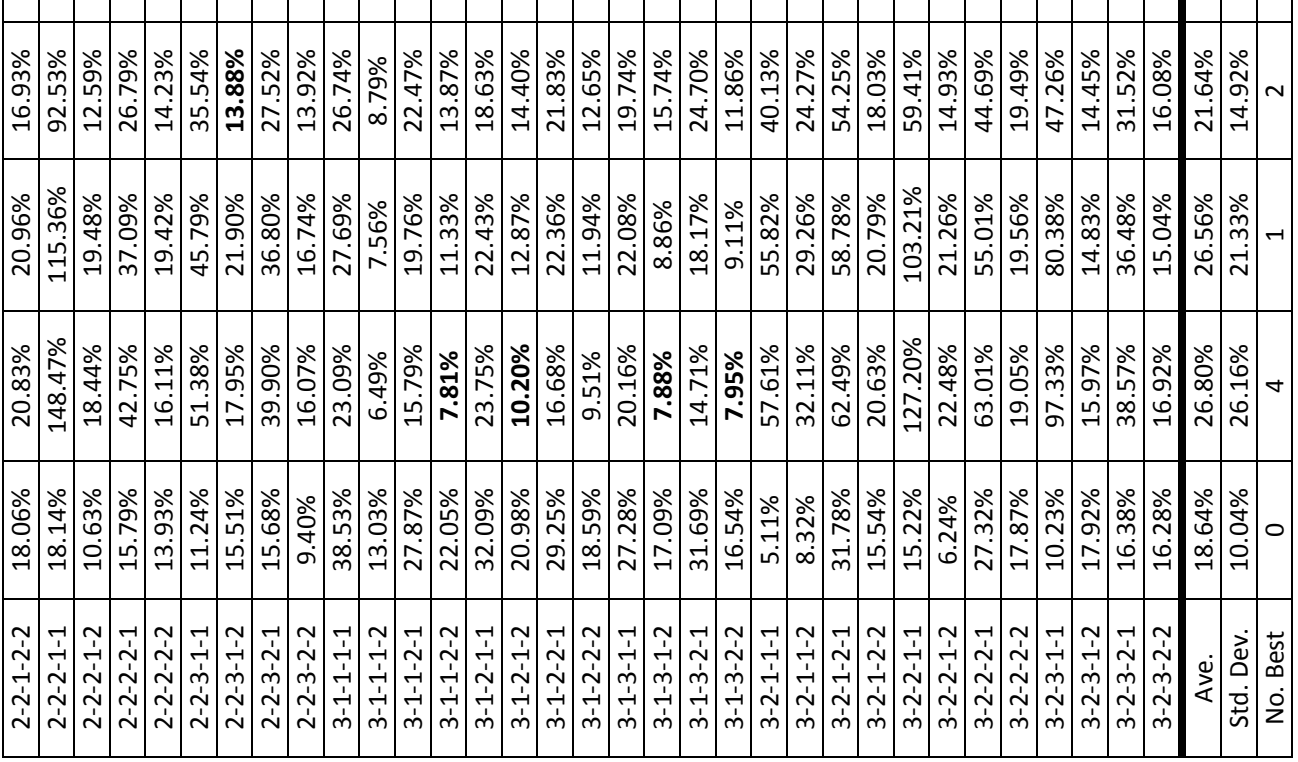




\section{References}

Boysen N, Briskorn D, Emde S (2015) A Decomposition Heuristic for the Twin Robots Scheduling Problem. Naval Res Logist, 62(1), 16-22. doi 10.1002/nav

Carlo HJ, Vis IFA (2009) New Initiatives in Stacking Cranes Configurations. Port Technology International 44: 32-36.

Carlo HJ, Vis IFA (2012) Sequencing Dynamic Storage Systems With Multiple Lifts and Shuttles. Int J Prod Econ 140: 844-853. doi: 10.1016/j.ijpe.2012.06.035

Carlo, HJ, Vis, IFA, Roodbergen, KJ (2014) Storage Yard Operations in Container Terminals: Literature Overview, Trends, and Research Directions, Eur J Oper Res, 235(2): 412-430. doi: 10.1016/j.ejor.2013.10.054

Dell RF, Royset JO, Zyngiridis I (2009) Optimizing Container Movements Using One and Two Automated Stacking Cranes. J Ind Manag Optim 5(2):285-302. doi: 10.3934/jimo.2009.5.285

Dorndorf U, Schneider F (2010) Scheduling Automated Triple Cross-over Stacking Cranes in a Container Yard. OR Spectrum 32:617-632. doi: 10.1007/s00291-010-0206-3

Gharehgozli AH, Laporte G, Yu Y, de Koster R (2015) Scheduling Twin Yard Cranes in a Container Block, Transportation Science, Articles in Advance, pp. 1-20. doi $10.1287 /$ trsc. 2014.0533

Erdogan G, Battarra M, Laporte G (2014) Scheduling twin robots on a line. Naval Res Logist 61(1), 119-130. doi: 10.1002/nav.21570

Gottwald (2015) ASC Automated Stacking Crane for the Port of Antwerp, Belgium. Accessed 4 February, 2015.

Li W, Yong W, Petering MEH, Goh M, de Souza R (2009) Discrete Time Model and Algorithms for Container Yard Crane Scheduling. Eur J Oper Res 198:165-172. doi: 10.1016/j.ejor.2008.08.019

Murty, KG, Liu, J, Wan, YW, \& Linn, R (2005) A Decision Support System for Operations in a Container Terminal. Decis Support Syst, 39, 309-332. doi:10.1016/j.dss.2003.11.002

Park T, Choe R, Ok SM, Ryu KR (2010). Real-time Scheduling for Twin RMGs in an Automated Container Yard. OR Spectrum 32(3):593-616. doi 10.1145/2245276.2245323

Vis IFA, Carlo HJ, Diaz P, Laboy ME, VanWijgaarden B (2010) Control Policies for a Dynamic Storage System with Multiple Lifts and Shuttles. In: Ellis KP, Gue KR, de Koster RBM, Meller RD, Montreuil B, Ogle MK (eds.) Progress in Material Handling Research, Material Handling Institute, Charlotte, North Carolina, pp 494-509 
Zhou W, Wu X (2009). An Efficient Optimal Solution of a Two-Crane Scheduling Problem. Asia-Pacific Journal of Operational Research 26(1), 31-58. doi:

10.1142/S0217595909002146 